\title{
CONF-971070--1
}

\section{General Formulation for Wavefront Curvature Correction in Polar-Formatted Spotlight-Mode SAR Images Using Space-Variant Post-Filtering}

\author{
N. E. Doren, Student Member, IEEE \\ C. V. Jakowatz, Jr., Member, IEEE \\ D. E. Wahl P. A. Thompson *
}

\begin{abstract}
In this paper, we introduce a general formulation for wavefront curvature correction in spotlight-mode $S A R$ images formed using the polar-formatting algorithm (PFA). This correction is achieved through the use of an efficient, image domain space-variant filter which is applied as a post-processing step to PFA. Wavefront curvature defocus effects occur in certain SAR collection modes that include imaging at close range, using low center frequency, and/or imaging very large scenes. Our formulation is general in that it corrects for wavefront curvature in broadside as well as squinted collection modes, with no computational penalty for correcting squint-mode images. Algorithms such as the range migration technique (also known as seismic migration), and a recent enhancement known as frequency domain replication, FReD, have been developed to accommodate these wavefront curvature effects. However, they exhibit no clear computational advantage over space-variant post-filtering in conjunction with polar formatting (PF2). This paper will present the basic concepts of the formulation, and will provide computer results demonstrating the capabilities of space-variant post-filtering.
\end{abstract}

\section{INTRODUCTION AND FORMULATION}

The classic approach to SAR image formation from phase history data collected in the spotlight-mode has been the polar-formatting algorithm (PFA). In polar formatting, the collected phase history data are described in terms of a slice of the three-dimensional Fourier transform of the scene reflectivity data, obtained on a polar raster [1-3]. An inverse Fourier transform of these data (as projected onto a chosen two-dimensional plane) forms the SAR image. The derivation of this technique relies upon the unrealistic assumption of strictly planar wavefronts in the transmitted microwave pulses. Any actual amount of curvature present in these wavefronts introduces two forms of distortion into the SAR image, as formed by the polar-format processor. First, there is a geometric distortion, which takes on the

*The authors are with the Sandia National Laboratories, MS0572, P. O. Box 5800, Albuquerque, NM, 87185. This work was supported by the US Department of Energy under contract No. DE-AC04-94AL85000. Sandia is a multiprogram laboratory operated by Sandia Corporation, a Lockheed Martin Company, for the United States Department of Energy.

form of a keystone ${ }^{1}$. This distortion can be rectified in a straightforward manner by appropriate post-warping of the image. The second form of distortion is a quadratic, spacevariant defocusing effect. The magnitude of this defocus effect is a function of the range and cross-range position of the target, and becomes greater for those targets placed further in range and cross-range from the scene center. This effect is not removable via post-warping, but instead requires space-variant refocusing of the formed image. The usual approach to this situation has been simply to limit the size of the scene reconstructed, so that the effects of wavefront curvature are not realized. That is, it can be shown that by limiting the reconstructed image size to

$$
L=\rho_{x} \sqrt{\frac{2 r_{0}}{\lambda}}
$$

where $L$ is the radius of the scene, $r_{0}$ is the range from the radar to the scene center, $\rho_{x}$ is the cross-range resolution, and $\lambda$ is the radar wavelength, the quadratic defocus effect for any target in the scene will be held to less than $\pi / 4$ radians, resulting in only a negligible amount of smearing ${ }^{2}$.

In this paper, we show that the computational burden involved in performing the space-variant restoration to remove the wavefront curvature defocusing effects is not particularly severe. For a certain set of imaging scenarios, it can be shown to be as small as $30 \%$ of the polarformat image formation time. The idea is to implement a space-variant image-domain filter, based upon an analytical derivation of the phase error that is induced by the curved wavefronts.

In [1], it is shown that the defocus effect of wavefront curvature on a spotlight-mode SAR image formed with polar formatting is a space-varying one that occurs in the cross-range (azimuth) direction only, at least for the condition wherein the radar is operating at a range that is large compared with the diameter of the scene reconstructed, and the collection is taken at broadside. Under this assumption, the Fourier transform of the blur function can be shown to be phase-only and is given by $^{3}$

$$
H(X) \approx \frac{x_{0}^{2}-y_{0}^{2}}{2 r_{0} k_{0}} X^{2}
$$

where $k_{0}=4 \pi / \lambda, r_{0}$ is the standoff range of the radar platform, $\left(x_{0}, y_{0}\right)$ is the location of a target projected into the

\footnotetext{
${ }^{I}$ See [1], pp. 361-363.

${ }^{2}$ See [1], pp. 95-97.

${ }^{3}$ See [1], pp. 361-363.
} 


\section{DISCLAMIER}

Portions of this document may be illegible in electronic image products. Images are produced from the best available original document. 


\section{DISCLAIMER}

This report was prepared as an account of work sponsored by an agency of the United States Government. Neither the United States Government nor any agency thereof, nor any of their employees, make any warranty, express or implied, or assumes any legal liability or responsibility for the accuracy, completeness, or usefulness of any information, apparatus, product, or process disclosed, or represents that its use would not infringe privately owned rights. Reference herein to any specific commercial product, process, or service by trade name, trademark, manufacturer, or otherwise does not necessarily constitute or imply its endorsement, recommendation, or favoring by the United States Government or any agency thereof. The views and opinions of authors expressed herein do not necessarily state or reflect those of the United States Government or any agency thereof. 
slant plane, and $X$ is the phase-history (Fourier transform domain) frequency associated with the cross-range image dimension.

In our general formulation for calculating quadratic phase error due to wavefront curvature, we remove the restriction that requires the operating range to be large compared to diameter of the scene reconstructed, thereby producing an exact solution for all standoff ranges and patch diameters. It is a general solution that takes into account not only broadside collection scenarios, but also squint-mode collections, where at mid-aperture, the radar platform is looking either backwards or forwards towards the center of the scene. This formulation is accomplished by first creating a geometric model of the imaged scene with respect to the radar's flight path, and subsequently deriving an equation for the phase return from an arbitrary point in the scene back to the radar platform. Next, the phase equation is represented as a polynomial via a two-dimensional Taylor series expansion. The linear, first order terms of this series represent the position of the target in the scene, and include residual terms that induce the keystone warping. The second order terms, known as the quadratic phase error terms, account for the nonlinear defocus, or wavefront curvature effect, of targets in the imaged scene. Higher order terms are ignored, since they are typically small in magnitude and have a negligible effect on the image distortion. Even for squint-mode collections, regardless of the degree of squint, all second order phase error terms reduce to zero, except the one associated with the cross-range (azimuth) direction. The value of this quadratic phase term is found to be

$$
\begin{aligned}
& H(X)= \\
& \frac{1}{2}\left\{\frac{1}{k_{0}}\left[2 r_{0}-r_{c}-\frac{r_{0}^{2}}{r_{c}}+\frac{x_{0}^{2} r_{0}^{2}}{r_{c}^{3}}\right]\right. \\
& +\frac{2 \tan (\theta) r_{0}}{r_{c} k_{0}}\left[x_{0}+\frac{x_{0} y_{0} r_{0}-x_{0} r_{0}^{2}}{r_{c}^{2}}\right] \\
& +\frac{\tan ^{2}(\theta) r_{0}}{r_{c} k_{0}}\left[2 r_{c}-3 r_{0}+2 y_{0}\right. \\
& \left.\left.+\frac{r_{0}^{3}-2 y_{0} r_{0}^{2}+y_{0}^{2} r_{0}}{r_{c}^{2}}\right]\right\} X^{2}
\end{aligned}
$$

where $r_{c} \approx r_{0}-y_{0}+1 / 2\left(x_{0}^{2} / r_{0}\right)$ is the distance from the target to the platform at mid-aperture, and $\theta$ is the squint angle at aperture midpoint. Thus, even for an exact solution that considers squint-mode collection scenarios, the filter required to remove quadratic phase error remains onedimensional, in the azimuth direction. This is significant in that the additional computational overhead associated with implementing a two-dimensional filter is completely avoided. If we again assume that the imaged patch size is much smaller than the standoff range, then equation (3) can be approximated by

$$
\begin{aligned}
& H(X) \approx \\
& \quad \frac{1}{2}\left\{\frac{x_{0}^{2}-y_{0}^{2}}{r_{0} k_{0}}-\tan (\theta)\left[\frac{2 x_{0} y_{0}}{r_{0} k_{0}}\right]-\tan ^{2}(\theta)\left[\frac{x_{0}^{2} y_{0}}{r_{0}^{2} k_{0}}\right]\right\} X^{2}
\end{aligned}
$$

where $x_{0}, y_{0} \ll r_{0}$. While this approximation may not be sufficiently accurate for extreme close-in collections, its simplified form gives us clearer insight into the effects of standoff range and squint angle on quadratic phase errors. Note that when $\theta=0$, this equation reduces to that of (2). That is, for a squint angle of zero, the general case reduces to the more specific case of a broadside collection. While each term of (4) contributes to the overall quadratic phase error, the sum of the terms is complicated in nature. For example, when $x_{0}=y_{0}$ for some arbitrary nonzero value of $x_{0}$, the first term of (4) contributes nothing to the error, while the next two terms contribute significantly, if $\theta \neq 0$. Conversely, when $x_{0}=0$, the first term contributes to the phase error for a nonzero $y_{0}$, while the next two terms contribute nothing. In practice, we have found that the peak quadratic phase error over an imaged scene is comparable between squinted and non-squinted collections, when all other parameters remain unchanged. However, it is quite apparent that the peak phase error increases inversely with the standoff range or radar center frequency. This increases the rate of change of the quadratic phase over the imaged patch, which tends to increase the computational burden. This increase is not significant except in extreme cases of close-in imaging or very low frequency radars.

\section{FILTER IMPLEMENTATION}

By applying an appropriate spatially-varying filter to the image that is formed by the polar-format processor, the defocus effects induced by (3) can be compensated. The filter kernel is constructed by calculating the quadratic phase error over the region of support covered by the filter, using the image location residing at the filter's center, $\left(x_{0}, y_{0}\right)$, as the reference point for calculating the error. This data is then conjugate multiplied, point by point, by the Fourier transform of the image data within the filtered region. This removes the quadratic phase error from that region of the scene, after which the data is inverse Fourier transformed back into the image domain. Alternatively, one may look at the process as a spatially-variant, image domain convolution mask, derived from the Fourier transform of the quadratic phase error function at that point in the scene. In some instances, spatial convolution may be more computationally efficient than using frequency domain filtering.

This filtering concept was first introduced in [4] as a method to remove phase errors in polar-formatted data that are directly Fourier transformed, without first resampling the data onto a rectangular grid. Unfortunately, while mathematically sound, this is a computationally burdensome method for removing polar format blurring because of the extreme amount of distortion present. However, as we will show, space-variant filtering is an effective way to remove the effects of wavefront curvature, which are generally much smaller than those of polar-format defocus. Space-variant filtering for wavefront curvature correction was first discussed in [5], but included only an approximation for broadside-mode wavefront curvature error. In this paper, we have presented the equation needed for exact calculation of quadratic wavefront curvature error, for both broadside and squint-mode cases at all standoff ranges.

The image is focused by moving the one-dimensional, space-variant filter across the image in the cross-range (azimuth) direction, for each line of range data. Each subse- 
quent filter overlaps the previous by some amount $c$, and has a filter length of $m$. Thus, the separation between adjacent filters, $s$, is $m-c$. If the filter were changed at every pixel, in strict accordance with the expression of (3), the computational burden associated with filter implementation could become excessive. Fortunately, the procedure can be made considerably more efficient than this by varying the filter function only as rapidly as required to maintain the residual defocus at an acceptable level.

Each filter operation exactly compensates for the phase error of the image pixel at the filter's center, but is only an approximation to the correction for points surrounding it. The approximation worsens toward the edges of the filter. Thus, the filter's length, $m$, which is one dimensional in the azimuth direction, is limited by the rate of change of the wavefront curvature error at that point in the image. In practice, the filter length should be sufficiently small to allow no more than $\pi / 4$ radians of phase error across the aperture after correction. This ensures that the IPR mainlobe is narrow enough to keep image defocus to within sub-pixel limits. Furthermore, the overlap between adjacent filters, $c$, must be sufficiently large to accommodate the support (width) of the defocus blur. The amount of overlap may be chosen larger or the filter length chosen smaller, without sacrificing image quality, if this helps to minimize the computational operations count, as shown in (5).

To further reduce the computational burden of wavefront curvature correction, a space-variant procedure for adjusting filter length and overlap can be implemented. The phase error at a point in the image is given by (3), and the instantaneous rate of change is given by its derivative. These parameters are used to determine the length and overlap of a uniformly sized and spaced post-filter, as well as the space-variant filter coefficients. Just as phase errors vary spatially, so do the requirements for filter length and overlap. While a worst-case filter length and overlap can be chosen which will correctly refocus all regions of the image, this is not computationally efficient. Instead, the filter length and overlap can be adjusted spatially, as is done with the filter coefficients, resulting in a significant reduction in computational burden when compared to a fixed filter length and overlap.

\section{B. Operations Count}

Each filter kernel of length $m$, where $m$ is typically a power of two, is multiplied, point by point, by the Fourier transform of the $m$ image data pixels at the filter point in the scene. The data are then inverse Fourier transformed. back into the image domain. In terms of complex multiplies, this takes $m \log _{2} m+m$ operations. Given a square image of $n \times n$ pixels, a filter overlap of $c$ pixels, and a separation of $s=m-c$ pixels, the overall operations count in terms of complex multiplies is found to be

$$
C_{p}=\frac{m n^{2}}{m-c}\left(\log _{2} m+1\right)
$$

The filter design puts a constraint on the maximum value of $s$, the kernel spacing, which is based on the extent of the quadratic defocus in the azimuth direction. Also, the filter kernel length, $m$, has a constraint on its maximum value, to ensure negligible residual defocus. However, as detailed in the previous section, $s$ can be further reduced, or $m$ reduced, while still obeying these constraints, in order to minimize the operations count in equation (5). Spacevariant overlap and kernel sizing can further reduce computation time.

\section{Computer Simulation Results of PF2}

Figure 1 illustrates the effect of wavefront curvature correction on a simulated point target lying near the edge of a patch. The parameters used for generation of the synthetic point target are shown in Table 1. For these parameters, note that (1) predicts that the maximum scene patch diameter that would be free of wavefront curvature defocus effects would be 381 meters. As a result, one would definitely expect to see degradations in targets placed near the extreme range and cross-range positions of the $1000 \mathrm{~m}$ scene. Indeed, the target in the left photograph of figure 1 exhibits nonlinear wavefront curvature distortion in the azimuth direction. The right photograph photo shows the same target after applying space-variant post-filtering. In this case, the filter size was chosen to be 32 (cross-range) pixels wide, based on evaluation of the maximum rate of variation of the quadratic phase error function in the crossrange direction. (As described in the previous section, the criterion used here is that the amount of quadratic phase should not be in error by more than $\pi / 4$ radians for any point inside the filter, so that we guarantee negligible residual defocus). The filter function was changed every 16 cross-range pixels, and on every range pixel. For this example, this overlap is sufficient to cover the extent of the blur in the cross-range direction. Figure 2 displays the corresponding IPR's for the target before and after range curvature correction.

\begin{tabular}{|l|l|l|}
\hline SAR parameter & symbol & value \\
\hline wavelength & $\lambda$ & $0.06 \mathrm{~m}$ \\
\hline cross-range resolution & $\rho_{x}$ & $0.33 \mathrm{~m}$ \\
\hline range resolution & $\rho_{y}$ & $0.33 \mathrm{~m}$ \\
\hline stand-off range & $r_{0}$ & $10,000 \mathrm{~m}$ \\
\hline image patch size & $D$ & $1000 \mathrm{~m}$ \\
\hline image size & $N$ & $4096 \times 4096$ pixels \\
\hline squint angle & $\theta_{s}$ & $26.8^{\circ}$ \\
\hline
\end{tabular}

Table 1: Parameters For Generation of Synthetic Target

Wavefront curvature processing time over the entire $4096 \times 4096$ pixel image was less than three minutes on a 200 $\mathrm{MHz}$ Sun Ultra workstation. This amounts to about $30 \%$ of the polar-format image formation time. For this imaging scenario, the entire PF2 computing time, including polarformat processing and the new space-variant post-filtering for wavefront curvature correction, was 569 seconds.

\section{Comparison of PF2 to Range Migration Processing}

Two range migration processing techniques were implemented in FORTRAN on a $200 \mathrm{MHz}$ Sun Ultra workstation and compared to the results obtained from the PF2 image formation algorithm in the previous section. The running times for these algorithms are listed in Table 2. 
The algorithms used for comparison are the range migration algorithm (RMA) [6], and a modified, more computationally efficient version of RMA known as FReD [7]. One of the drawbacks of the RMA algorithm is that it requires the phase history data to be upsampled in the along-track dimension at a rate that depends upon the collection geometry. ${ }^{4}$ For some imaging scenarios, such as those involving significant squint angles, the time to compute the upsampled data may be quite large. The FReD algorithm was developed to avoid the upsampling requirements, and hence render migration processing reasonably efficient in these situations. For the case simulated here, the required upsampling ratio for RMA was approximately 2:1.

\begin{tabular}{|l|l|l|}
\hline Algorithm & $\begin{array}{l}\text { Execution } \\
\text { time (sec.) }\end{array}$ & $\begin{array}{l}\text { Collection } \\
\text { Geometry }\end{array}$ \\
\hline $\begin{array}{l}\text { PF2 (fixed filter } \\
\text { overlap and width) }\end{array}$ & 569 & $\begin{array}{l}\text { broadside or } \\
\text { squinted }\left(26.8^{\circ} \text { ) }\right.\end{array}$ \\
\hline RMA & 1153 & broadside \\
\hline FReD & 713 & broadside \\
\hline
\end{tabular}

Table 2: Execution Times for Three Algorithms in FORTRAN on a SUN Ultra 2 Workstation

\section{Conclusions and Suggested Future Work}

Overall, space-variant post-filtering for wavefront curvature removal is a computationally efficient, straightforward extension of the traditional polar-format algorithm. It provides an exact solution to the wavefront curvature problem for all standoff distances and patch sizes, and is effective for squinted as well as broadside data collections. It does not require subaperture processing and the associated "patchwork" reassembly of the image, nor is it iterative or have any dependence on the visual contents of the scene. Thus, the new polar-formatting with post-filtering algorithm (PF2) should be considered as a viable candidate for a spotlight-mode image formation processor when wavefront curvature effects are present.

Our FORTRAN implementation of the new algorithm, PF2, would indicate that it is more computationally efficient than the original version of the seismic migration technique, RMA, and even more efficient than the migration processing version known as $\mathrm{FReD}$, that avoids the need for along-track upsampling. However, FORTRAN timings are not the final word on algorithm efficiency, especially in situations such as this, where algorithms are not necessarily tuned to their maximum computational efficiency. A careful operations count needs to be performed to further investigate the relative computational merits of these techniques. Furthermore, the question of how the range migration techniques can be applied to spotlight-mode SAR image collection geometries other than broadside needs to be more thoroughly studied. RMA becomes problematic at large squint angles, because the along-track upsampling demands under these conditions is severe. Also, it is not clear at this point whether or not RMA or FReD can be applied to non-straight line collections. A comparison of these, as well as other algorithms that can accommodate wavefront curvature, such as the class of techniques known as "subaperture processing," should be conducted to cover realistic imaging modalities.

\footnotetext{
${ }^{4}$ See [6], pp. $480-481$ and $487-489$.
}
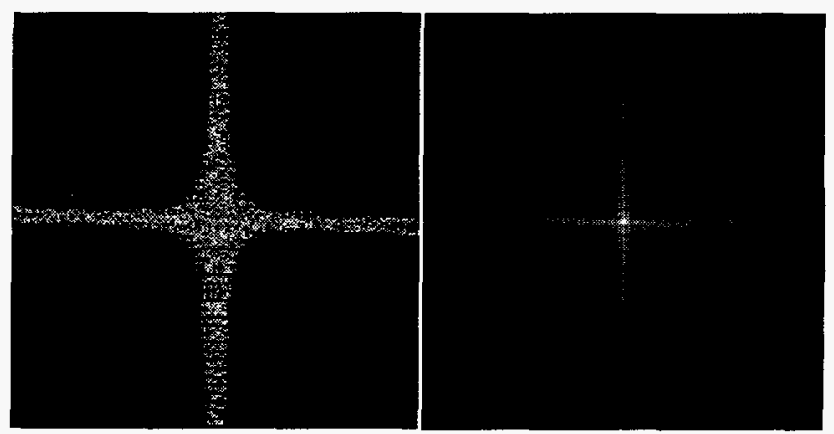

Figure 1: Point Targets Before/After Correction

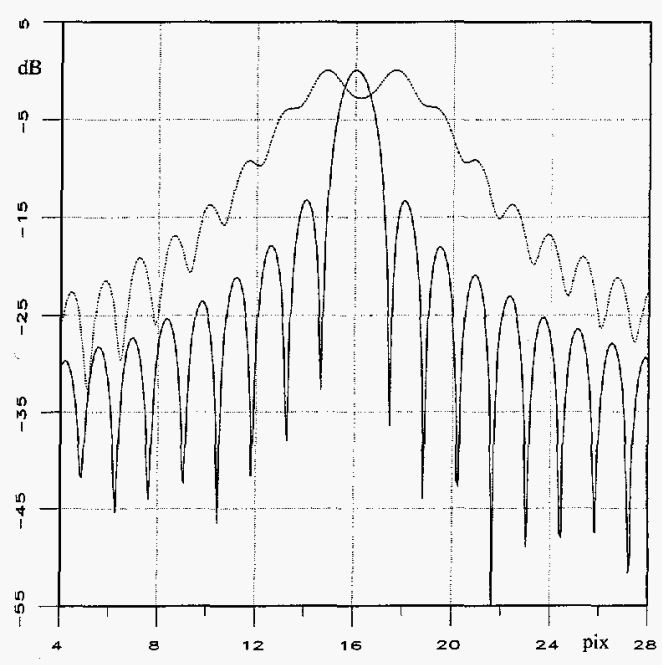

Figure 2: Point Target IPRs Before/After Correction

\section{REFERENCES}

[1] Jakowatz, Charles, et. al., Spotlight-Mode Synthetic Aperture Radar: A Signal Processing Approach, Kluwer Academic Publishers, Boston, 1996.

[2] Walker, J. L., "Range-Doppler imaging of rotating objects", IEEE Trans. AES-16, 1980, pp. 23-52.

[3] Asherman, D. A., et. al., "Developments in radar imaging", IEEE Trans. AES-20, 1984, pp 363-400.

[4] Kong, K. K. and Edwards, J. A., "Polar format blurring in ISAR imaging", IEE Electronic Letters Online. No: 19950998, 15 June 1995.

[5] Jakowatz, C. V. et al., "Wavefront curvature correction in spotlight-mode SAR images using space-variant postfiltering", SPIE 11th Annual Int. Symp. Aerospace Defense Sensing and Controls Conference, 21 April 1997.

[6] Carrara, W. et al., Spotlight Synthetic Aperture Radar: Signal Processing Algorithms, Artech House, Boston, 1995.

[7] Golden, A. et al., "Migration processing of spotlight SAR data", Proc. SPIE Symp. Algorithms for SAR Imagery, Orlando, FL, April 1994. 